\title{
The Construction of the "Tour-factor Integration" Teaching Quality Monitoring System in Higher Vocational Colleges - Jiangyin Polytechnic College is taken as an example
}

\author{
Qingtang ZHANG \\ Jiangyin Polytechnic College, \\ Jiangyin 214405, Jiangsu, China
}

\author{
Chengying SHEN \\ Jiangyin Polytechnic College, \\ Jiangyin 214405, Jiangsu, China
}

\begin{abstract}
Teaching quality is the lifeline of higher vocational colleges, but also the foundation of education and development. In this paper, on the basis of analyzing teaching quality and the concept of teaching quality monitoring and combining the practice of Jiangyin Polytechnic College, the methods and problems in the construction of the teaching quality monitoring system of higher vocational colleges are discussed.
\end{abstract}

Keywords: Higher Vocational Colleges; Teaching Quality; Monitoring System

\section{Introduction}

Making a comprehensive improvement to the education and teaching quality is a requirement clearly raised in the Planning Outline for the National Medium and Long-term Education Reform and Development (2010-2020) and the Decision of the State Council on accelerating the Development of Modern Vocational Education, but also a necessary way for the survival and development of higher vocational colleges. Higher vocational colleges must adhere to the concept of integrated production and education and the concept of school-enterprise cooperation, and actively construct a scientific, rational, practical and efficient teaching quality monitoring system from four aspects (i.e. top design, standard setting-up, dynamic evaluation, and bidirectional interaction) around the new meaning and requirement of regional economic and social development for training skill-oriented personnel, and give full play to the role in supervision, regulation, guide and motivation. Thus, the awareness of the front-line teachers and teaching management personnel in quality, efficiency and the local economic and social development service can be improved continually, and then the improvement of college teaching quality and the promotion of the fit and matching degree between talent training and social demand can be achieved with great efforts.

\section{Teaching quality and its monitoring}

Teaching quality is the degree of the teaching task and training goal achieved by school according to the teaching objective, which is a reflection to the overall quality of school education [1]. Teaching quality monitoring is a process of certain institutions and personnel to monitor and adjust all parts and factors possibly affecting teaching quality according to certain specifications and standards so as to realize the school educational objective and personnel training goal [2].

\section{The current situation of teaching quality monitoring}

At present, all higher vocational colleges have preliminarily established a scientific and reasonable teaching quality monitoring system under the guidance of dean's office or supervisor office and the cooperation of affiliated teaching and research offices. Meanwhile, in order to 
guarantee the normal operation of the teaching quality monitoring system, higher vocational colleges have spent a lot of energy on the internal management. For example, many institutions and personnel have been arranged, and many rules and regulations have been formulated by all departments. Along with the constant advancement of the higher vocational education reform, many problems in the teaching quality monitoring of higher vocational colleges have gradually emerged, and the problem about what to monitor, who to monitor and how to monitor has been especially obvious.

\section{The strategies for the construction of teaching quality monitoring system}

To build a scientific, reasonable, practical and efficient teaching quality monitoring system, higher vocational colleges must pay attention to the following respects.

\subsection{Top design: Establishing a scientific teaching quality target system}

Top design should be a professional engineering term, and its basic meaning is to find the sources by hard and thorough search, assume overall responsibility, and consider all levels and elements of a project, and then define the solutions to problems at the highest level.

Using the total quality management theory in management, the teaching quality monitoring system in higher vocational colleges should consist of beforehand-stage, mid-stage, and post-stage, and can be generally divided into four aspects (i.e. objective system, standard system, operation system, and organization system).

\subsection{Standard setting-up: Establishing a complete teaching quality standard system}

To build a complete teaching quality standard system, higher vocational colleges can use the principle of the total quality management, actively carry out the related education teaching reform requirements from the higher levels, tightly emphasize all key parts of the teaching process, establish a sound teaching management system of rules and regulations, and timely formulate and perfect the quality standards of Professional Talent Training Scheme.

\subsection{Dynamic assessment: Implementing strict teaching quality monitoring}

In order to the effectiveness of teaching quality monitoring, higher vocational colleges can draw lessons from Jiangyin Polytechnic College and actively pay attention to five key aspects. First, it is necessary to focus on the personnel training scheme. By hiring outside-school experts to provide informal discussions and conducting questionnaire surveys and interviews among graduates, higher vocational colleges can know well the needs of employing units, the comments on college graduates and the suggestions about college's personnel training. Then, they can get an understanding of graduates' opinions about the major personnel training model and objective, course system, teaching contents, teaching methods and other aspects, in order to provide a scientific reference basis for further improving the professional personnel training scheme. Second, it is necessary to focus on the classroom teaching quality. The inspection and supervision on the classroom teaching quality must be strengthened, so as to find the problems, provide feedback in time, promote the advanced teaching experience, and help great numbers of teachers improve the classroom teaching quality. Third, it is necessary to focus on the special supervision and inspection. Supervisor office not only implements the regular supervision tasks such as teaching supervision, and attending a class and evaluation classroom teaching, but simultaneously takes part in special supervision activities with functional departments such as the dean's office, student affairs office, and admission office in major construction, curriculum construction, teaching material construction, teaching and research section construction, and school-enterprise cooperation, 
field work, tracking graduates and outside-school practice, for the purpose of realizing a virtuous cycle of monitoring, feedback, and improvement. Fourth, it is necessary to focus on the institutions. A teaching committee guided by school president can be established, but the daily work can be managed by vice President who is in charge of teaching. The dean's office, as a functional department, plans the teaching management of school as a whole. Supervision office is responsible for supervising and inspecting the daily teaching. The corresponding teaching management institutions can be established by all departments. Two-level monitoring system composed by college and departments can be implemented, and the dean's office and the supervision office have their own duties and can mutually cooperate and support each other in the process of teaching monitoring and evaluation, in order to carry out a top-level monitoring for all teaching parts. All departments are the education entities implementing the personnel training and teaching quality monitoring and evaluation, and they undertake the second-level monitoring of teaching quality. Fifth, it is necessary to focus on the improvement of system. Higher vocational colleges must improve all related teaching management systems and quality standards and let them implemented in all aspects (e.g. teaching plan, operation, quality, and basic construction) of teaching, practice teaching management and teaching staff management, so as to monitor and manage the teaching quality as a whole.

\subsection{Bidirectional interaction: Paying attention to the information feedback of teaching quality monitoring}

PDCA is also called as quality loop, which a universal model in management. In 1930, it was first proposed. In 1950, Deming, American quality management expert, mined PDCA again and extensively applied to constantly improving the process of product quality. Total quality management activities constantly and repeatedly operate in a whole process according to PDCA.
PDCA includes four stages and eight steps, as shown in Fig.1 [4].

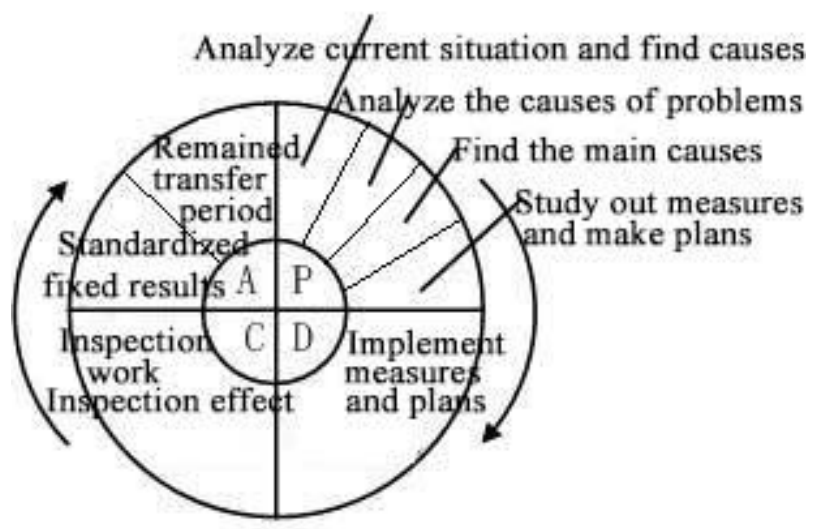

Fig.1 the four stages and eight steps of PDCA

Generally speaking, the main contents in information feedback are the completions of the teaching quality objectives and standards, and the effectiveness of the concrete implementation of the teaching quality monitoring system. Information collection and feedback ways are shown as follows. First, written report is applied. The dean's office or the supervision office can analyze the quality problems in the main teaching parts and generate a research report to feed back to the leaders of college and departments, major functional divisions, and teachers. Second, special meetings are held. Teaching inspection feedback meeting must be held timely after a special teaching inspection is completed. Third, all kinds of informal discussions are conducted. Colleges can regularly conduct student representative and employing unit representative symposiums every semester in order to know well the teaching dynamics of all departments, collect the opinions and suggestions of students and employing units, and timely provide feedback and rectification. Fourth, brief feedback is provided. The dean's office or the supervision office can timely classify and edit the opinions and suggestions of students about the teaching into brochure at the end of each month and then give feedback to the leaders of college, departments, and main functional divisions in a form of brief report, making it easier for related departments and personnel to make an 
improvement in a targeted way. Fifth, special network channels are set up. Special channels can be established on campus network, and special personnel are arranged to receive the opinions and suggestions of teachers and students about the teaching quality monitoring and timely provide answers and feedback.

\section{Points necessary to focus on in the construction of the teaching quality monitoring system}

Constructing a teaching quality monitoring system is a serious and complicated task, in which higher vocational colleges must pay attention to dealing with several points as follows.

First, it is necessary to focus on the interests of all related parties. The purpose of teaching quality monitoring is to ultimately determine the results achieved by the school teaching quality according to the needs and value orientation of sponsors, students and society. Therefore, a good teaching quality monitoring system must be based on the wide participation and full support of all related parties and also adheres to the principle of mutual benefit and win-win results.

Second, it is necessary to focus on the integrated use of information means. In the process of teaching quality monitoring, the Internet technologies, various information technology means and the advantages of cloud computing and big data analysis must be applied fully. For example, higher vocational colleges can intensively integrate and transfer information by combining the personnel training work state data acquisition platform for the sake of multi-channel information collection, and then regularly analyze and report it and discover the main factors possibly affecting the teaching quality. As a result, they can strengthen the pertinence, effectiveness and efficiency of the teaching quality monitoring.

Third, it is necessary to focus on the construction of harmonious campus culture. In the construction of teaching monitoring system, higher vocational colleges should pay attention to the construction of harmonious campus culture and give full play to the functions such as demonstrative leading role, cohesion, and spur motivation of campus culture [5].

\section{Conclusion}

The construction of teaching quality monitoring system is a complicated system project for higher vocational colleges, and it needs constant exploration and innovation. The construction of teaching quality monitoring system using the concept of "tour-factor integration" is a kind of beneficial exploration, which is helpful for higher vocational colleges to know well the idea of construction, scientifically set up the quality control objective system and standard system, implement teaching quality monitoring, effectively arouse the enthusiasm of all parties, and promote the constant increase of teaching quality and the degree of match between personnel training and social needs.

\section{Acknowledgement:}

This paper aided financially by the Research Project of the Higher Education Educational Reform of Jiangyin Polytechnic College in 2014 (2014-JG-XZ-01).

\section{References}

[1] Baowen HAO. Study on Teaching Quality Concept and Generated Model [J]. Journal of Inner Mongolia Normal University (Philosophy and Social Sciences Edition), 1995 (4).

[2] Ruizhi ZHANG. The Exploration and Practice of the Undergraduate Teaching Quality Monitoring Long-term Mechanism in Colleges and Universities [J]. Journal of Education Theory and Practice, 2014 (5).

[3] Wen-tao REN. Consideration on the Higher School Teaching Quality Monitoring and 
Evaluation System [J]. Journal of Education Theory and Practice, 2007 (8).

[4] Minyan CAI, Genji CAO. The Construction of the Teaching Quality Guarantee System in
Higher Vocational Colleges [J]. Journal of Higher Vocational Education, 2014 (25). 